\title{
Pilot Drone for Convoy Escort
}

\author{
U.Saravana kumar, P.Ilangovan, R.Rakesh, S.Sathish Rakin
}

\begin{abstract}
Escort is an exceedingly difficult and consequential task. The pilot vehicle can only detect the problem and traffic on the road. In recent years attackers started using the drone to strike down the leaders from a long distance. For such problem obstacles on the way and through Air, and also Attack like drone strike cannot be predicted by the human escorts accurately even before or at the time during escorting. The paper aims to make a PILOT DRONE to detect unauthorized air activity, traffic, and obstacles. It facilitates the escorts to monitor the Realtime status with a high-speed pilot drone. Captured data will be transferred to the ground station immediately. So that the status will be monitored continuously. A quadcopter with a size of $220 \mathrm{~mm}$ is used in this work to satisfy the requirements, such as speed, flight time and lightweight to carry from one place to another. This proposed kit will help to protect leaders from airstrikes and also used to improve the safety of leaders and VIP.
\end{abstract}

Keywords: Drone, Quadcopter, Squad, Escort, Pilot drone.

\section{INTRODUCTION}

DRONE (Dynamic remotely operated navigation equipment) is an unmanned aerial vehicle that is used for civil and military purposes. In India, it is mainly used for Aerial photography and videography. A quadrotor is generally propelled by using symmetrically pitched blades. The system is fixed with four rotors and four ESC. Quadcopters are easy to carry and easy to use in open grounds. They are controlled and lifted by the guidance of the flight controller and onboard computer. It creates a vertical thrust for take-off and landing. The less weight design of frame thrust required is lower and it results in saving battery capacity.Normal Quadcopter will have a size of $450 \mathrm{~mm}$ to $800 \mathrm{~mm}$ also fixed with lower $\mathrm{KV}$ motors for creating thrust through which we cannot achieve high speed. So it is planned to develop a new system with a 220mm frame and higher $\mathrm{kV}$ motors, it achieves the speed of $90 \mathrm{~km} / \mathrm{hr}$.

Manuscript published on November 30, 2019.

* Correspondence Author

U.Saravana kumar*, Assistant Professor with the Department of Electronics and Communication Engineering, Periyar Maniammai Institute of Science and Technology Vallam, Thanjavur, India,

P.Ilangovan, Assistant Professor with the Department of Compute Science and Engineering, Periyar Maniammai Institute of Science and Technology.

R.Rakesh, AssistantProfessor with the Department of Electronics and Communication Engineering, Periyar Maniammai Institute of Science and Technology.

S.Sathish Rakin, Engineering student in the Department of Electronics and communication engineering at Periyar Maniammai institute of science and technology vallam, Thanjavur.

(C) The Authors. Published by Blue Eyes Intelligence Engineering and Sciences Publication (BEIESP). This is an open access article under the CC-BY-NC-ND license http://creativecommons.org/licenses/by-nc-nd/4.0/
As the size of the frame is small and less in weight it has low air resistance and can respond in the speed of 32khz.The hardware architecture supports to decrease air resistance. Due to its small structure, it can easily travel in opposite wind direction with absolute stability. larger quadcopters cannot move fast in the opposite wind because of its structure, it is designed only for carrying payload and not for racings. In order to attain speed specifications, the

quadcopter components are considered. when we use the small size frame, it gives great speed with powerful processing.Quadrotors are defined as mechanical devices that can assist the escort's teams continuously in any situation. This is the next step in developing the technology towards safety and surveillance.

\section{METHODOLOGY}

\section{A. Quadcopter definition}

The quadcopter is one of the types of multirotor which is lifted by the help of four motors and propellers fixed in the clockwise and counter-clockwise direction. It can perform (VTOL) [15] vertical take-off and land in any weather condition and in uneven places. The quadcopter can move in all directions in a stabilized condition, by changing the speed of four motors separately via ESC electronic speed controller. ESC consists of MOSFET (Metal oxide semiconductor field-effect transistor) which controls the speed of each and every motor based on the signal received from the flight controller. This signal is generated by the value received from the gyroscope present in the flight controller.

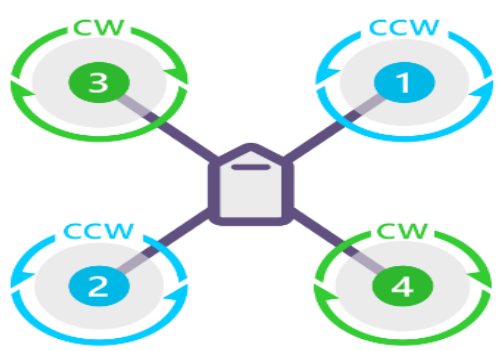

\section{QUAD $X$}

\section{Fig.1. Motor configuration}

A quadcopter is propelled and lifted by its two pairs of motors. Diagonal motor spins in the same direction. The reason diagonal motors are spinning in the same direction is to eliminate yaw motion occurring on the quadcopter so that it can fly in a stabilized condition [6] [7].

\section{B. Operating techniques}

$>$ There are three axes to define the point of stability of the quadcopter [8] [9]. Shown in Figure 2.

$>$ Each axis contains its unique Gyroscope.

$>$ Three-Axis

$\checkmark$ Pitch

$\checkmark$ Roll

$\checkmark$ Yaw 


\section{* Pitch}

This operation is achieved by increasing the speed in one of its rear side motors.

* Roll

This operation is achieved by increasing the speed in one of its side motors.

* Yaw

This operation is achieved by increasing the speed in diagonal motors.

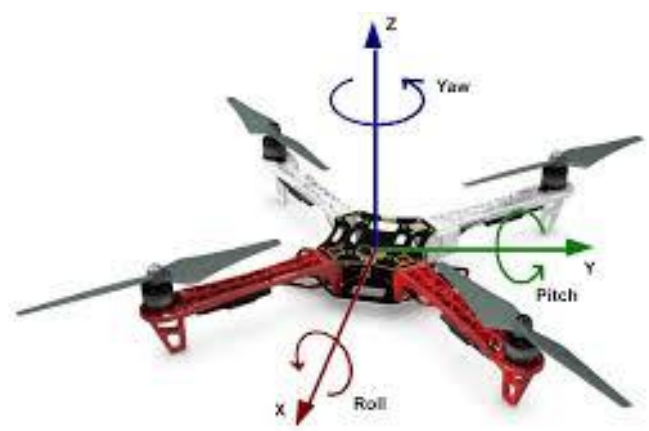

Fig.2. Three-axis of the quadcopter

\section{Quadcopter components}

\section{1) Frame}

The frame of the quadcopter is very important for stability and to eliminate resistivity while flying in the air and also it should be suitable for placing all the mechanical and electronic components. It is made up of carbon fiber. Carbon fiber provides vibration-free flight and also provides the quadcopter to be weightless. Shown in Fig3.

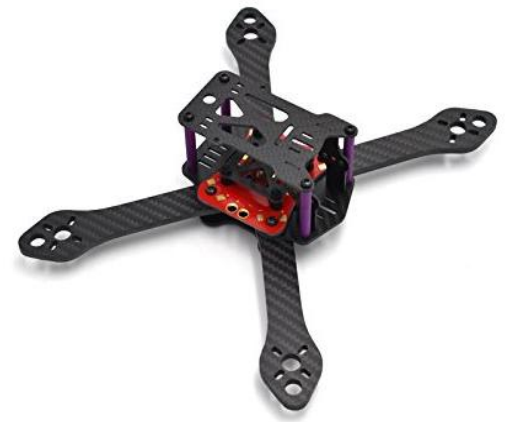

Fig.3. carbon fiber frame

2) Motor

The motor used in this Quadcopter is a brushless dc motor which is an out-runner motor. The speed of the motor is defined by Kilovolt. Here the armature winding act as a stator and permanent magnet act as a rotor of the motor. With the help of electrical power, commutation is done without brushes. This brushless motor provides high speed to the quadcopter and has high dynamic performance [4]. As shown in Figure 4.

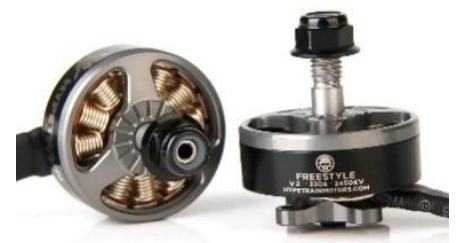

Fig.4. Motor

3) Electronic Speed Controller (ESC)

The electronic speed controller (ESC) controls the speed of the Brushless dc motor. By using the signal given from the Flight controller. It consists of the Metal Oxide
Semiconductor Field Effect Transistor (MOSFET) when the switching frequency has changed the speed of the motor will change using this the whole Quadcopter is controlled and stabilized during flying in the air. As shown in Figure 5.

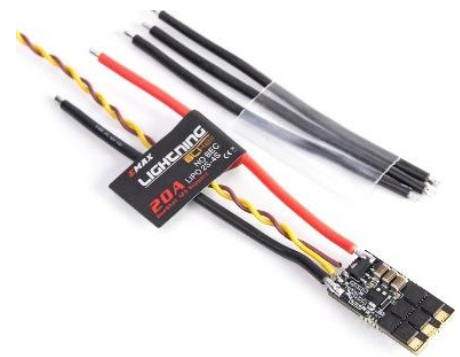

Fig.5.Electronic Speed controller

4) The flight controller (FC)

The flight controller is the brain of the quadcopter. It receives data from the receiver, sensors, and the data is transferred to the main processor to process the raw data after processing the data is converted and stored. All these processes are achieved within a second. It uses powerful F405 MPU with the combination of High-speed 20602 gyros with an advanced filtering system [12]. Shown in Figure 6.

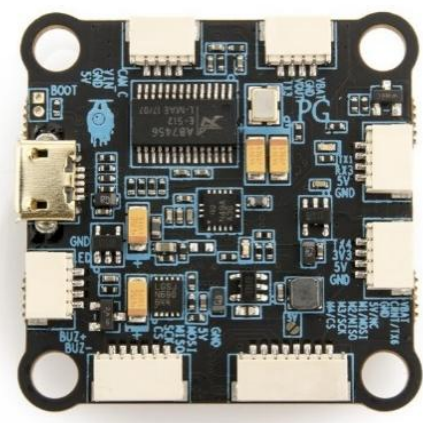

Fig.6.Flight Controller

\section{Light Imaging Detection and Ranging}

LIDAR (Light Imaging Detection and Ranging) is a module which is used for mapping in Drones as the new implementation of this lidar one can retrieve the activity in air through the pilot drone. It takes the laser ranging from 8000 samples at high speed. Setting up this in the vertical and horizontal direction we can generate the ground and air data. $10 \mathrm{hz}$ is the typical scanning frequency of this lidar range finder.it can also be adjusted from a 5hz-15hz range based on the requirement of the scanning area. As shown in Figure 7.

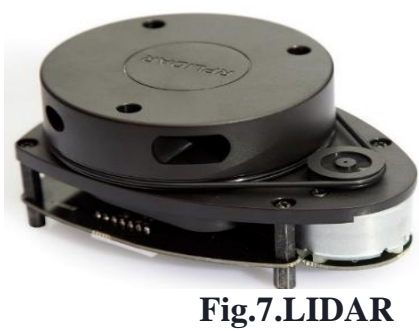

\section{E. LIDAR Mechanism and working}

Lidar uses the pulsed laser in the form of light to measure the range. It creates precise and accurate information on the scanned surface. There is an embedded DSP, starts processing the sample data. This whole setup is split up into the range scanner system and motor system. As shown in Figure 8. This motor system is used to spin the laser in 360 degrees.

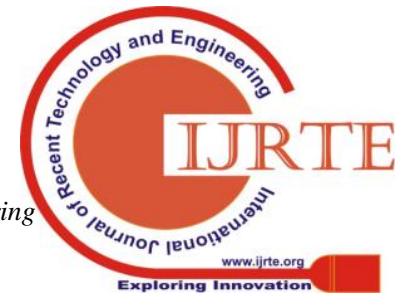


The operating voltage of the motor system is $5 \mathrm{v}$ dc, and the motor spins in the clockwise direction. To communicate with the range finder, we have to use either USB or UART interfaces with the setup [1] [2].

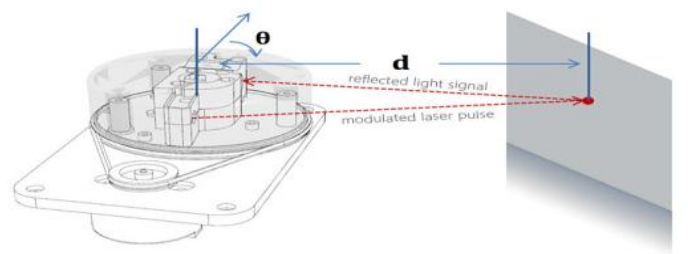

Fig.8. Working of LIDAR

F. Block diagram of the proposed system

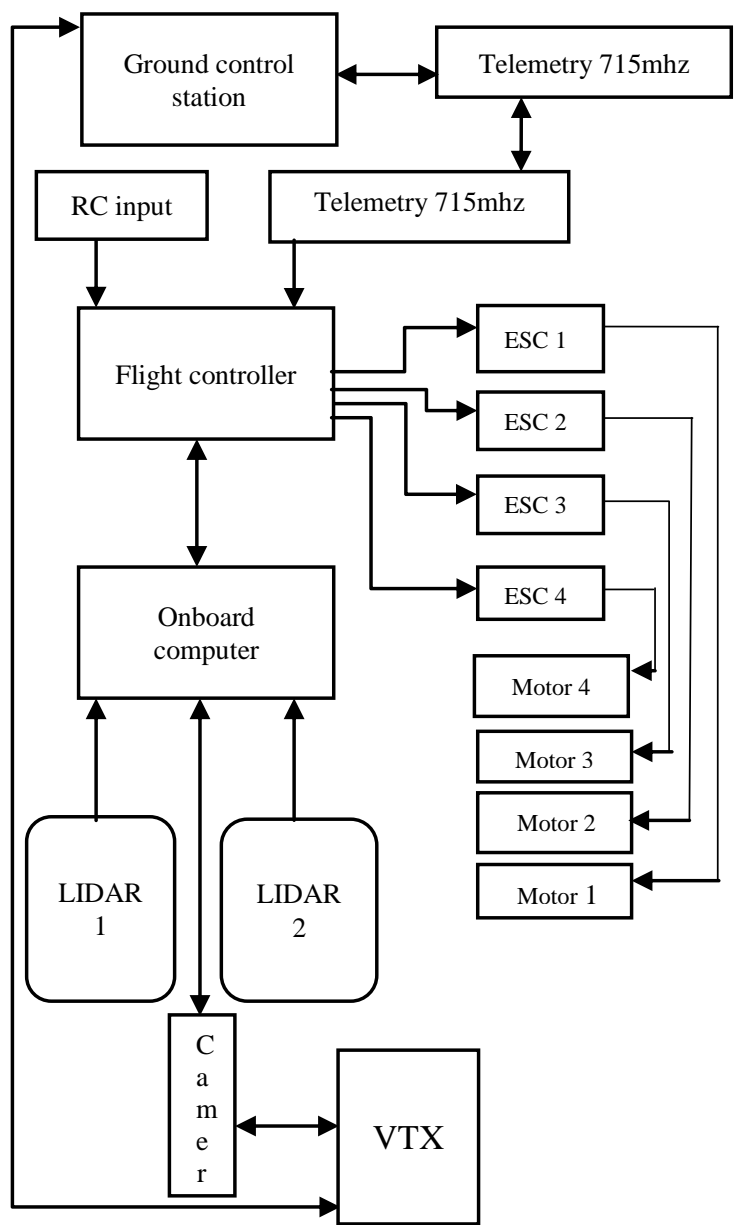

Fig.9.Block diagram of whole Pilot drone setup with ground station control

Figure.9 shows that there are two main controllers used in the pilot drone and also two transmitters are used for data transferring and live video transfer. Both the antenna operates in two different signal bands. Data transferring system operated in $2.4 \mathrm{Ghz}$ and the video telemetry used a 5.8 Ghz system for distortion less communication.

In the above block diagram, the lidar is connected to an onboard computer and with the flight controller Also we can receive the scanned data either via onboard computer or through the cloud and telemetry receiver.

\section{IMPLEMENTATION}

The proposed system was implemented and assembled in two different stages
Stage 1 - In this stage, the design and components selection are done for the quadcopter with suitable electronics and mechanical parts. As a result of stage 1 can be seen in fig. 10 .

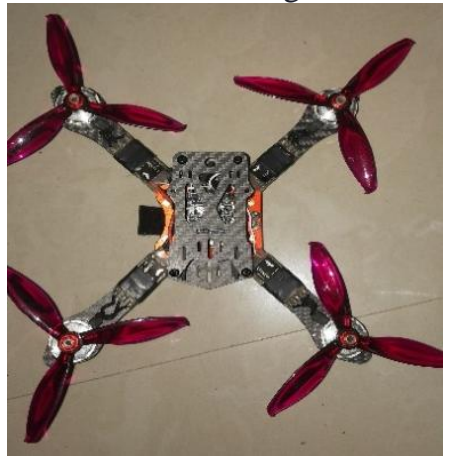

Fig.10.Hardware implementation top view.

Stage2 - Here the design, development and assembly of the scanning system is done with LIDAR and Onboard computer i.e. Raspberry pi. It acts as a Processor for the LIDAR system, which is integrated with the flight controller through the serial communication port. Another Lidar is directly connected to the flight controller via the UART port. As the LIDAR cannot be connected directly to the Raspberry pi; we used the ULN2003A IC it acts as a driver circuit for the LIDAR. It achieves high amplification power through Dc coupled bipolar transistors. Shown in Figure 11.

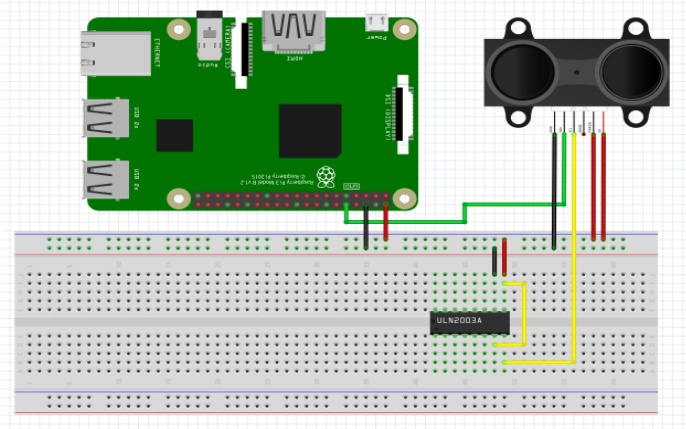

Fig.11 LIDAR integration.

\section{TESTING \& RESULT}

Testing of the PILOT DRONE for Convoy Escort is done in two stages of experiments.

Experiment 1(Flight test)

Experiment 1 is conducted to analyze the flight time of the quadcopter with the battery capacity of 2200 mah and also to compute the stability of the system in normal wind speed and in high wind. All these criteria are compared with the $450 \mathrm{~mm}$ drone to bring out the best result.

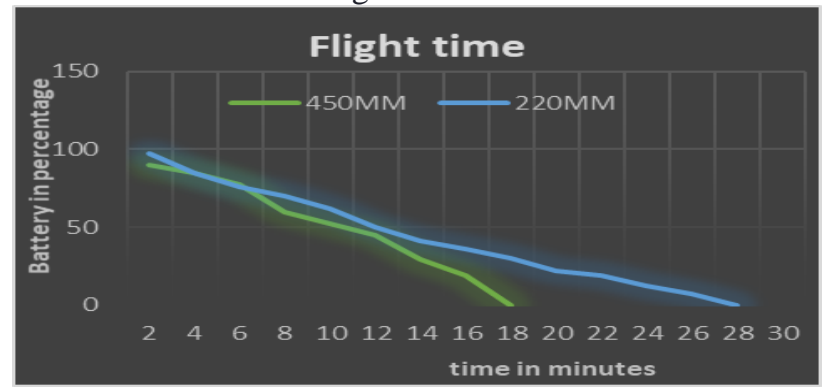

Fig.12. Flight time of our system with the comparison of $450 \mathrm{~mm}$ drone. 


\section{Experiment 2 (LIDAR test)}

This experiment is conducted to test the lidar system in the environment and to obtain the processed data from the PILOT DRONE to the ground Station.

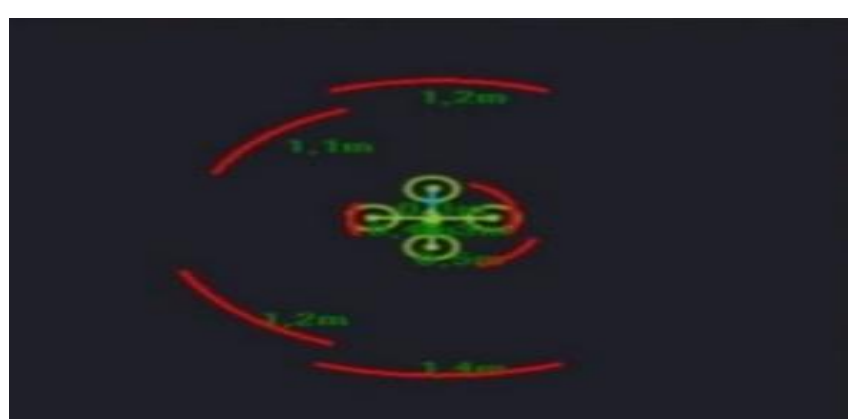

Fig.13. Data from the flight controller's LIDAR.

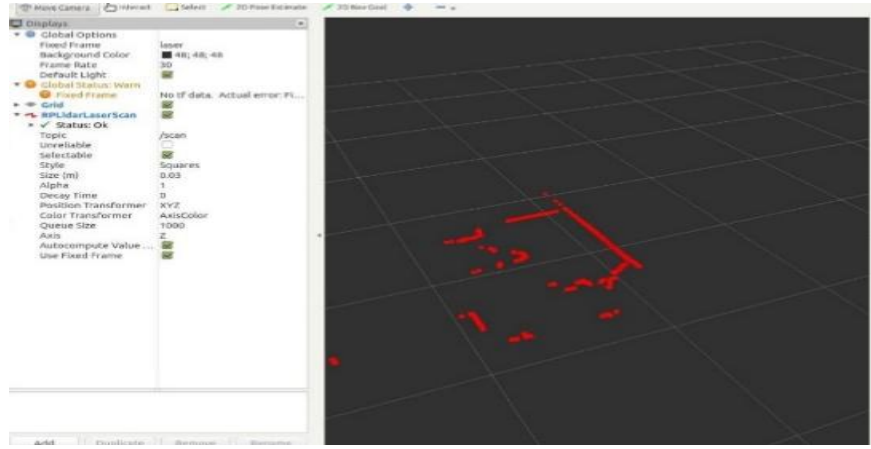

Fig.14. Data from the Raspberry pi.

These above outputs are obtained from the LIDAR sensor. Figure 13 shows the AIR data, figure 14 shows the ground-level data. This figure shows that this pilot drone can perform in both criteria simultaneously without any distortions in the flight time.

In Fig.14 the output is obtained through the lidar sensor connected with Raspberry pi. here the scanned data is first passed to an IC ULN2003A to amplify the signal. Because the output from the sensor is weak to process to the ground station so the output is amplified and the processed data is transferred to the Raspberry pi. Using this data a 3D map can also be created but the drone is traveling at high speed hence the required data for $3 \mathrm{~d}$ mapping is insufficient.

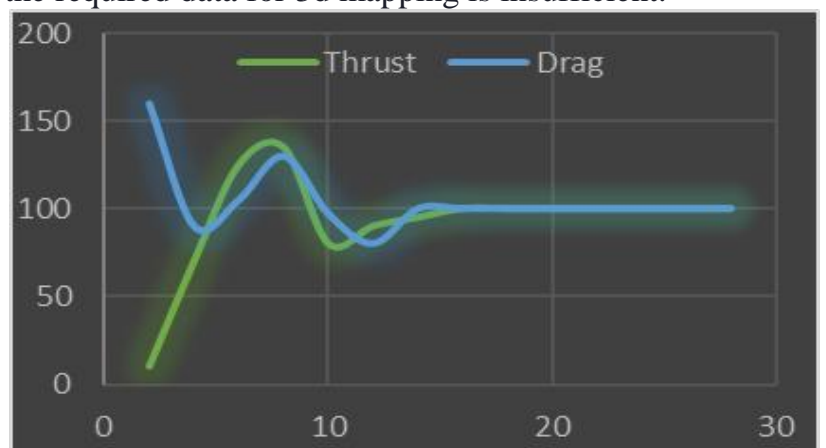

Fig.15 Thrust and drag created by quadcopter.

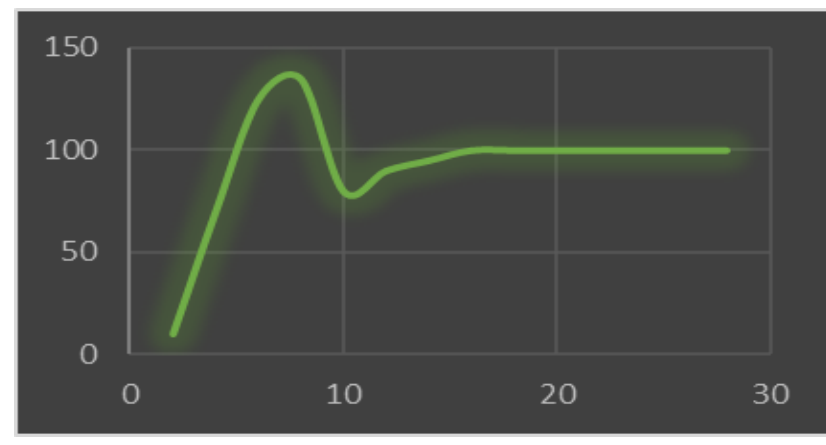

Fig.16. Actual Thrust.

\section{CONCLUSION}

Pilot Drone for Convoy Escort has been designed and implemented using the $220 \mathrm{~mm}$ carbon fiber frame with the LIDAR sensor on both Horizontal and Vertical directions. The scanned and measured raw data are analysed and processed by using the Digital signal processor which is already inbuild in the Integrated circuit.

Based on the experiment, this system is found perfectly suitable for using in real-time surveillance for Convoy Escort. It also helps to improve security and minimize the risk involved in the safety of leaders. As an extension of this research work, we have several ideas have been formulated to improve the Pilot drone for future use. also, the high-end lidar sensor has to be used for improving precision. Moreover, from the experiment done here delivers that the study of this concept will pave way for improving the safety of leaders.

\section{REFERENCES}

1. Deilamsalehy, H., \& Havens, T. C. (2016). Sensor fused three-dimensional localization using IMU, camera, and LiDAR. 2016 IEEE SENSORS. DOI:10.1109/icsens.2016.7808523

2. A Chambers, S Scherer, L Yoder, S Jain, S Nuske, S. Singh, "Robust multi-sensor fusion for micro aerial vehicle navigation in GPS-degraded/denied environments", American Control Conference (ACC) 2014

3. Alzu'bi, H., Akinsanya, O., Kaja, N., Mansour, I., \& Rawashdeh, O. (2015). Evaluation of an aerial quadcopter power-plant for underwater operation. 2015 10th International Symposium on Mechatronics and Its Applications(ISMA). DOI:10.1109/isma.2015.7373488

4. Lee, T.-Y., Seo, M.-K., Kim, Y.-J., \& Jung, S.-Y.(2016). Motor Design and Characteristics Comparison of Outer-Rotor-Type BLDC Motor and BLAC Motor Based on Numerical Analysis. IEEE Transactions on Applied Superconductivity, 26(4), 1-6. DOI:10.1109/tasc.2016.2548079

5. D.Domingos, G. Camargo and F. Gomide, "Autonomous Fuzzy Control and Navigation of Quadcopter," in Science Direct, 2016.

6. V. Thanh and H. Tung, "Optimizing P.I.D Parameters in Control Accelerometers And Gyroscopes In Self-Balancing Quadrotors," Journal of Science and Technology, p. Vol. 1, 2015

7. A. O'Dwyer, Handbook of PI and PID Controller Tuning Rules, Imperial College Press, 2nd edition, 2009.

8. A. Suresh, M. V. Kiran and C. S. Kumar, "DesignPID controller for TITO process based on least square optimization tuning method," 2014 IEEE International Conference on Computational Intelligence and Computing Research, Coimbatore, 2014, pp. 1-4.

9. Y.Wakasa, K. Tanaka, and Y. Nishimura, "Online controller tuning via FRIT and recursive least-squares," in Proceedings of the IFAC Conference on Advances in PID Control (PID '12), 2012.

10. A. S. Silveira, A. A. R. Coelho, and F. J. Gomes, "Model-free adaptive PID controllers applied to the Benchmark PID'12," in Proceedings of the 2nd IFAC Conference on Advances in PID Control (PID '12), Brescia, Italy, March 2012. 
11. R. Ranganayakulu, G. U.B. Babu and A. S. Rao, "Fractional filter IMCPID controller design for an unstable inverted pendulum system," 2017 IEEE International Conference on Smart Technologies and Management for Computing, Communication, Controls, Energy and Materials (ICSTM), Chennai, 2017.

12. Long, H. and Chung, Y. (2016).Design of the Nonlinear Structure Adaptive Model Inversion Flight Control System. Journal of Robotics and Mechatronics, 28(6).

13. K.N.Maleki, K.Ashenayi, L. R. Hook, J. G. Fuller, and N. Hutchins, "A reliable system design for non-deterministic adaptive controllers in small UAV autopilots," 2016 IEEE/AIAA 35th Digital Avionics Systems Conference(DASC), Sacramento, CA, 2016.

14. Muhammed Iqbal Fadholi, Priyo Sidik Sasingko, Suhartono, Sutikno," Autonomous Pole Balancing Design In Quadcopter Using Behaviour-Based Intelligent Fuzzy Control” 2018 2nd conference on informatics and computational sciences (ICICoS), Indonesia.

15. Jong Tai Jang, Sanghyuck Han, "Analisis for VTOL flight software of px4" $201818^{\text {th }}$ conference on control, automation and systems(ICCAS).

\section{AUTHORS PROFILE}

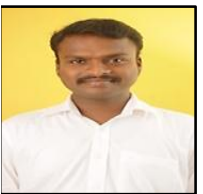

Mr. U.Saravana Kumar, Assistant Professo with the Department of Electronics and Communication Engineering, Periyar Maniammai Institute of Science and Technology Vallam, Thanjavur, India, has graduated in

Electrical and Electronics Engineering from Anjalai Ammal Mahalingam Engineering College (AAMEC) is a college in Thiruvarur District, Tamil Nadu in 2004 and took his post graduate degree on M.E Embedded System Technologies at College of Engineering, Guindy, Anna University, Chennai in 2009.Doing his Ph. D degree on Electrical and Electronics Engineering from National Institute of Technology, Trichirapalli.His area of interest is Embedded systems and Electric powered vehicle.

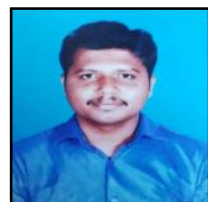

Mr.Rakesh.R received the B. Tech. degree in Electronics and Communication engineering from Mount Zion College of Engineering, Pudukkottai affiliated to Anna University, Chennai, India in 2005, and M.E. degree in Industrial Engineering from College of Engineering, Anna University, Chennai, India in 2007. He is pursuing the Ph.D. degree in Robotics Engineering with the Centre for Automation and Robotics in Hindustan Institute of Science and Technology,Chennai . He is currently an AssistantProfessor with the Department of Electronics and Communication Engineering, Periyar Maniammai Institute of Science and Technology.

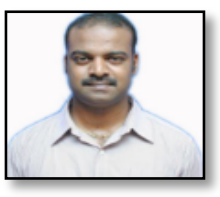

Mr.P.Ilangovan received the $B$. Tech. degree in Computer Science and Engineering from Bharathiyar College of Engineering and technology, Karaikal, Affiliated to Pondicherry University and M.E. degree in Periyar Maniammai University,Thanjavur, He is pursuing the

Ph.D. degree in Computer Science and Engineering in Periyar Maniammai Institute of Science \& Technology, Thanjavur. He is currently an Assistant Professor with the Department of Computer Science and Engineering, Periyar Maniammai Institute of Science and Technology.

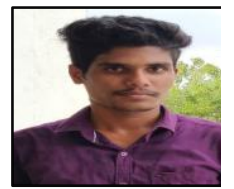

Mr.S.Sathish Rakin is an Engineering student in the Department of Electronics and communication engineering at Periyar Maniammai institute of science and technology vallam, Thanjavur. His area of interest is in the fields of drones and robotics. 